\title{
Post-operative respiratory failure due to bilateral phrenic nerve palsy
}

\author{
R.W. Burgess, A.F. Boyd, P.G. Moore and G.S. Oldfield
}

Royal Newcastle Hospital, Newcastle 2300, Australia.

\begin{abstract}
Summary: A 65 year old female patient developed a large left pleural effusion and a sternal split dehiscence following aorto-coronary artery bypass grafting. A second operation was performed to investigate and drain the pleural effusion and to repair the sternum. Subsequent to this operation the patient was in acute respiratory failure due to bilateral phrenic palsy. It is probable that the left phrenic nerve was damaged in the initial operation and the right nerve in the second. The patient's subsequent progress is described.
\end{abstract}

\section{Introduction}

Respiratory failure is a well recognized complication following thoracic surgery. Bilateral phrenic nerve palsy is a rare cause of postoperative respiratory failure. We report a case of respiratory failure due to bilateral phrenic nerve palsy which occurred subsequent to two thoracic procedures separated by three months.

\section{Case report}

A 65 year old female presented for repair of a sternal split dehiscence and investigation of a large left pleural effusion. She had undergone aorto-coronary artery bypass grafting, with saphenous vein and left internal mammary artery graft three months previously, for relief of a long history of ischaemic heart disease. Topical cardiac hypothermia in the form of pericardial ice slush was not used. Myocardial protection was afforded by use of systemic hypothermia to $26^{\circ} \mathrm{C}$ and cold cardioplegia introduced into the aortic root. The procedure had been uneventful and the patient was discharged home after 10 days with relief of her symptoms of ischaemic heart disease. At home she could sleep well in a supine position but became easily fatigued and short of breath with minimal exertion. On review by her cardiologist a large left pleural effusion and a separation of the sternal split were noted. The skin incision had healed with no evidence of infection. A third heart sound and elevated jugular venous pressure to $4 \mathrm{~cm}$ above the manubriosternal

\footnotetext{
Correspondence: R.W. Burgess, F.F.A.R.A.C.S., Department of Anaesthesia, Newcastle Mater Misericordiae Hospital, Waratah, N.S.W. 2298, Australia

Accepted: 5 July 1988
}

junction were detected. Electrocardiogram showed widespread ST segment depression and $T$ wave changes. These features were consistent with cardiac failure due to post-operative pericarditis. Treatment with indomethacin and anti-cardiac failure therapy allowed resolution of the signs of cardiac failure but the left pleural effusion remained. Surgery was planned in order to drain the pleural effusion and repair the sternal dehiscence.

On presentation for surgery the patient was normotensive, weighed $60 \mathrm{~kg}$ and apart from the chest findings, physical examination was normal. She was mildly breathless at rest but there was no evidence of cardiac failure. Surgery consisted of an initial rigid bronchoscopy, to exclude bronchial obstruction of the left lower lobe, and a small amount of secretions was aspirated. A median sternal incision was then made, demonstrating that several of the sternal wires had broken and the remainder had cut through the sternum. There was moderate pericardial inflammation, and the pleura from both sides were adherent to the posterior aspect of the sternum. On entering the left hemithorax, $700 \mathrm{ml}$ of turbid fluid were drained. This fluid was later shown to have a high white cell and red blood cell count but was sterile on culture. The left lower lobe was collapsed but re-expanded with surgical manipulation and several sustained inspirations.

Whilst mobilizing the pleura from the posterior sternum to enable safe rewiring the right pleural space was accidently entered. The right brachiocephalic vein was inadvertently damaged during mobilization of the pleura from behind the manubrium and was successfully repaired with 6/0 Prolene after application of two Debakey clamps to control bleeding. Bilateral chest drains were placed, the sternum rewired and the chest closed. The sternal wiring was achieved by first placing

(C) The Fellowship of Postgraduate Medicine, 1989 
wires around the cartilages parallel to the sternal edge and then placing wires around these and the sternum to approximate the sternal edges. The anaesthetic, using a single lumen Portex endotracheal tube, was uneventful. The patient remained intubated and was transferred to the intensive care unit for positive pressure ventilation. A left subclavian central venous line was placed for management of fluid therapy. The following day the patient had normal oxygenation on controlled ventilation, with an $\mathrm{FiO}_{2}$ of 0.3 , was fully awake, co-operative and did not have excessive pain. Her muscle power was normal. On attempting to wean the patient off ventilation, she could only manage a vital capacity of $200 \mathrm{ml}$ and rapidly fatigued. The patient remained dependent on positive pressure ventilation, but in all other respects was satisfactory. Six days later, paradoxical abdominal movement was noted when the patient breathed spontaneously, whilst on intermittent mandatory ventilation. This suggested the possibility of diaphragmatic paralysis. This was investigated by fluoroscopic screening when paradoxical movements of both hemi-diaphragms were demonstrated. Percutaneous phrenic nerve conduction studies were performed, with electromyographic recordings over each hemi-diaphragm. This demonstrated no conduction on the left side and very limited response on the right side. With an amplitude of $0.06 \mathrm{mV}$ (normal $>0.4 \mathrm{mV}$ ) the findings on the right may have been due to a cutaneously conducted impulse.

A diagnosis of bilateral phrenic nerve palsy was made. This was supported by measurement of vital capacity and maximal breathing capacity in the lying, sitting and standing positions at 350,650 and $1100 \mathrm{ml}$ and $10.0,20.8$ and $27.6 \mathrm{l} / \mathrm{min}$, respectively.

A tracheostomy was performed and the patient remained on positive pressure ventilation when sitting or supine. Six weeks after surgery the patient could walk normally around the hospital, but became extremely short of breath within a few minutes of lying down and hypercapnia was demonstrated. Ten weeks after surgery she was able to sleep through the night in a sitting position without ventilation. A sleep study was performed monitoring haemoglobin/oxygen saturation with pulse oximetry and electroencephalography. This demonstrated that haemoglobin saturation was well maintained at greater than $90 \%$ saturation except in the rapid eye movement (REM) phase of sleep when it fell to $78 \%$. The patient did not waken and the saturation rapidly improved once non-REM sleep occurred. Repeat screening of the diaphragm still demonstrated bilateral paradoxical diaphragmatic movement. However, the patient's condition slowly improved and 11 weeks after surgery the patient was discharged. Three months after discharge the patient could perform housework, and walk one mile without stopping, and she could sleep in a semi-sitting position. Repeat phrenic nerve conduction studies demonstrated significant improvement in conduction on the left but no conduction on the right.

\section{Discussion}

Phrenic nerve palsy following cardiac surgery has been frequently reported especially following the use of iced saline slush in the pericardial sac. ${ }^{1-4}$ However, paralysis may occur without the use of topical cardiac hypothermia and the left phrenic nerve is more at risk. ${ }^{2,4}$ Unilateral paresis may give minimal symptoms but bilateral paresis can result in chronic ventilatory insufficiency and severe orthopnoea.

In this case report the patient's clinical course after the cardiac surgery was consistent with left phrenic nerve paresis. Paresis of this type can be expected to improve within about one year. Although it is not certain, it is likely that the right phrenic nerve may have been damaged in the second operation as it lies in proximity to the right brachiocephalic vein. Had this been noted at surgery the possibility of nerve repair could have been considered. ${ }^{5}$

The diagnosis of bilateral phrenic nerve palsy is frequently delayed ${ }^{3,4}$ due to a low level of clinical suspicion. Clinical hints which point to the diagnosis include paradoxical abdominal movement with respiration, orthopnoea in the absence of cardiac or respiratory disease, difficulty in weaning from assisted ventilation and lower lobe atelectasis.

The traditional 'sniff test' to confirm the diagnosis using fluoroscopy may be impossible to perform in the ventilated unco-operative patient and can produce false positive or negative results. ${ }^{3,6-8}$ The 'sniff test' relies on cephalad movement of the hemi-diaphragm on the paralysed side. ${ }^{9}$ Improved methods of confirming the diagnosis include transdiaphragmatic pressure studies, ${ }^{6}$ and phrenic nerve conduction studies using diaphragmatic electromyography, ${ }^{7}$ fluoroscopy or ultrasound ${ }^{8} \mathrm{~A}$ high index of suspicion must be maintained if delays in diagnosis are to be avoided. 


\section{References}

1. Scannell, J. - Discussion by McGoon, D., Mankin, H. \& Kirklin, J. Results of open heart operation for acquired aortic valve disease. J Thorac Cardiovasc Surg 1963, 45: 47-66.

2. Roussou, J.A., Parker, T., Engelman, R.M. \& Breyer, R.H. Phrenic nerve paresis associated with the use of iced slush and the cooling jacket for topical hypothermia. $J$ Thorac Cardiovasc Surg 1985, 89: 921-925.

3. Kohorst, W.R., Schonfeld, S.A. \& Attman, M. Bilateral diaphragmatic paralysis following topical cardiac hypothermia. Chest 1984, 85: 65-68.

4. Dajee, A., Pellegrini, J., Cooper, G. \& Karlson, K. Phrenic nerve palsy after topical cardiac hypothermia. Int Surg 1983, 68: 345-348.

5. Merav, A.D., Attai, L.A. \& Condit, D.D. Successful repair of a transected phrenic nerve with restoration of diaphragmatic function. Chest 1983, 84: 5: 642-644.
6. Brown, K.A., Hoffstein, V. \& Byrick, R.J. Bedside diagnosis of bilateral diaphragmatic paralysis in the ventilator - dependent patient after open heart surgery. Anaesth Analg 1985, 64: 1208-1210.

7. Moorthy, S.S., Markland, O.N., Mahomed, Y. \& Brown, J.W. Electrophysiologic evaluation of phrenic nerves in severe respiratory insufficiency requiring mechanical ventilation. Chest 1985, 88: 211-214.

8. McCauley, R.G. \& Labib, K.B. Diaphragmatic paralysis evaluated by phrenic nerve stimulation during fluoroscopy or real-time ultrasound. Radiology 1984, 153: 33-36.

9. Guenter, C.A. \& Whitelaw, W.A. The role of diaphragm function in disease. Arch Intern Med 1979, 139: 806-808. 